\title{
A STOCHASTIC MODEL FOR VERY HIGH RESOLUTION SAR AMPLITUDE IMAGES OF URBAN AREAS
}

\author{
Gerardo Di Martino, Antonio Iodice, Daniele Riccio, Giuseppe Ruello
}

Università di Napoli Federico II, Via Claudio 21, 80125, Napoli, Italy

\begin{abstract}
For the new generation of spaceborne SAR sensors a resolution even better than one meter in the spotlight acquisition mode can be obtained. However, due to the involved electromagnetic mechanisms dictating SAR image formation, direct interpretation of very high-resolution (VHR) SAR images is not straightforward. In this paper, we propose the use of the power spectral density (PSD) for the analysis and interpretation of VHR SAR images of urban areas. In particular, we introduce a theoretical model of the PSD of such images, and then we test the validity of the developed model on one COSMO/SkyMed spotlight SAR image of the area of Naples, Italy. Finally, we show how the parameters of the obtained PSD can be related to meaningful physical observables of the imaged urban scene.
\end{abstract}

Index Terms - SAR, urban areas, spectral estimation

\section{INTRODUCTION}

In recent years, the launch of COSMO/SkyMed and TerraSAR-X missions has made very high-resolution (VHR) SAR images of urban areas routinely available: in particular, a resolution even better than one meter can be achieved by COSMO/SkyMed SAR images acquired in the spotlight mode. In principle, from such images a huge amount of information about objects present in the urban scenario can be potentially extracted. However, the significant presence of geometrical distortions, such as layover, shadowing, multiple reflections, and other involved electromagnetic scattering phenomena significantly hampers direct interpretation of VHR SAR images of urban areas [1], [2].

In this paper, we investigate the use of the power spectral density (PSD) of VHR SAR amplitude images of urban areas for information extraction. As a matter of fact, a theoretical model for the PSD of SAR amplitude images of urban areas is presently not available and it is certainly not easy to predict the PSD of these SAR images, which are the result of an involved combination of single scattering from terrain and buildings, and multiple scattering from dihedral and trihedral structures. In particular, while for moderate- and lowresolution systems frequently all these scattering contributions are simultaneously present in a single resolution cell, for VHR SAR systems, such as
COSMO/SkyMed in the spotlight acquisition mode, the resolution cell is so small that dihedral and trihedral returns dominate with respect to the single scattering background. Accordingly, a realistic description of VHR SAR amplitude images can be obtained by considering sparse brilliant points, or lines, over a dark background: the positions of these brilliant points and lines can be considered randomly distributed, unless they belong to a building façade, in which case an ordered, periodic spatial distribution is expected [3], [4].

In the following section, we introduce a theoretical model based on the abovementioned concepts, while in Section 3 we show the results regarding one actual COSMO/SkyMed enhanced spotlight SAR amplitude image of the area of Naples, Italy. In addition, in Section 4, we provide hints on the exploitation of parameters of the obtained PSD to extract useful information from VHR SAR amplitude (or intensity) images of urban areas.

\section{THEORETICAL MODEL}

In view of the considerations reported above, we can assume that the amplitude (or intensity) image is a superposition of sparse brilliant points and lines, so that an image range cut $i(r)$ can be expressed as a superposition of $N$ pulses of width $\delta_{i}$ :

$$
i(r)=\sum_{i=1}^{N} A_{i} p_{i}\left(\frac{r-r_{i}}{\delta_{i}}\right)
$$

In case of randomly distributed pulse positions $r_{i}$, which is a reasonable assumption if no building façade is included in the considered portion of the SAR image, we can assume that $r_{i}$ are mutually independent random variables. In this case, the obtained PSD is an averaged version of the square modulus of the pulse Fourier transform and, in particular, if the different pulses have all the same shape and width, then the image cut PSD is directly proportional to the square modulus of the pulse Fourier transform:

$$
S(\eta)=\sum_{i=1}^{N}<A_{i}^{2} \delta_{i}^{2}\left|P_{i}\left(\delta_{i} \eta\right)\right|^{2}>.
$$


If the considered portion of the image includes a building façade, then $M$ out of the $N$ pulses are regularly spaced and they form a periodic pattern:

$$
i(r)=\sum_{i=1}^{N-M} A_{i} p_{i}\left(\frac{r-r_{i}}{\delta_{i}}\right)+\sum_{m=0}^{M-1} A_{0} p_{0}\left(\frac{r-r_{0}-m d}{\delta_{0}}\right) .
$$

The regular range spacing $d$ of the façade structure can be related to the building inter-floor distance via the SAR incidence angle [3]. In this case we can show that the obtained PSD is composed of two terms:

$S(\eta)=\sum_{i=1}^{N-M}<A_{i}^{2} \delta_{i}^{2}\left|P_{i}\left(\delta_{i} \eta\right)\right|^{2}>+<A_{0}^{2} \delta_{0}^{2}\left|P_{0}\left(\delta_{0} \eta\right)\right|^{2}>\left|\frac{\sin (\eta d M / 2)}{\sin (\eta d / 2)}\right|^{2}$

where the first one is an averaged version of the square modulus of the pulse Fourier transform, whereas the second one has peaks in correspondence of spatial frequencies $\eta_{n}=n(2 \pi / d)$.

A short discussion is now in order with regard to the pulse shape. On a SAR amplitude image, if we assume ideal processing and if a single pulse is present that corresponds to a pointlike scatterer (for instance, a trihedral), then its shape is the absolute value of a sinc function. More in general, for a non-ideal processing and/or if the pulse corresponds to a distributed scatterer with range size of the order of the range resolution (or, maybe, a group of very close pointlike scatterers), then the pulse shape can significantly depart from the absolute value of a sinc function. In particular, some simulations, and some experimental evidence (see Section 3) seem to indicate that a good approximation of the pulse shape is often given in this case by a Lorentzian function. It can be shown that, in this case, VHR SAR images of urban areas have an exponential spectrum: accordingly, the plot of the spectrum is a straight line in a linear-log plane. In Fig. 1 an example of plots of the PSD computed according to the proposed model for Lorentzian pulses are reported.

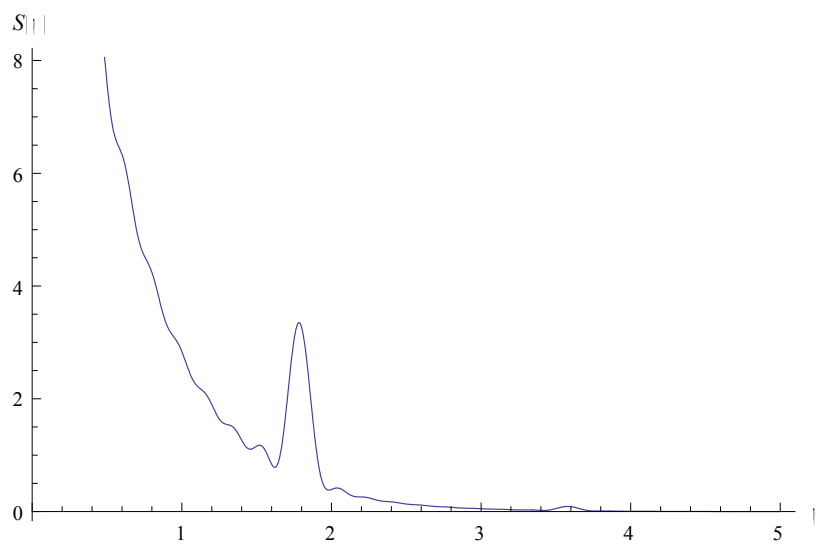

(a)

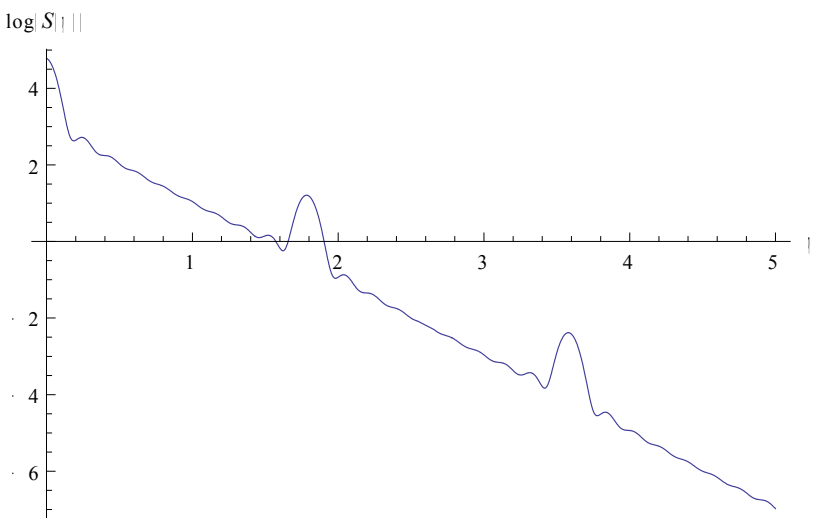

(b)

Fig.1 Linear (a) and logarithmic (b) plots of the PSD predicted by the proposed model for $\delta_{i}=1 \mathrm{~m}, d=3.5 \mathrm{~m}$, with $N=30, M=10$.

\section{EXPERIMENTAL RESULTS}

The proposed model has been verified using a set of enhanced spotlight Cosmo-SkyMed images of Naples, Italy, presenting different look angles. As an example, we show the results regarding an image acquired with a look angle of $44^{\circ}$, presenting a resolution of $1.07 \mathrm{~m} \times 0.679 \mathrm{~m}$ in azimuth-slant range geometry and a pixel spacing of $0.7 \mathrm{~m} \mathrm{x} 0.4 \mathrm{~m}$. On the image we selected three subsets: the first one is relevant to a scene made up essentially of old or historical buildings located in the area of the Forcella neighborhood; the second one is relative to an area pertaining to the business district of Naples enclosing several very tall buildings; the last one is the layover area of one single skyscraper, i.e. the tallest of the buildings forming the courthouse of Naples. In Fig. 2 we show a subset of the image including the three areas of interest, which are marked in green, blue, and red, respectively. For each subset, we compute the PSDs of the range profiles (using the Capon estimator [5]) and average them.

In Fig. 3 we report the three obtained plots of the PSDs. The plot in Fig. 3 (a) is relevant to the area of Forcella, which consists essentially of historical buildings presenting a small number of floors; moreover, these buildings are not oriented along the sensor line of flight. This implies the presence on the scene of a limited number of pulses. In this case, the obtained results are quite similar to the Fourier transform of the absolute value of the sinc function.

The plot in Fig. 3 (b) is relevant to the business district area. In this case, the tall modern buildings present on the scene are mainly oriented along the sensor line of flight and are responsible for the presence of a large number of bright points and lines on the image (and hence high pulses in the profiles). The graphs are better approximated by a straight line.

The PSD relevant to the case of the courthouse is reported in Fig. 3 (c). The presence of spectral peaks due to 


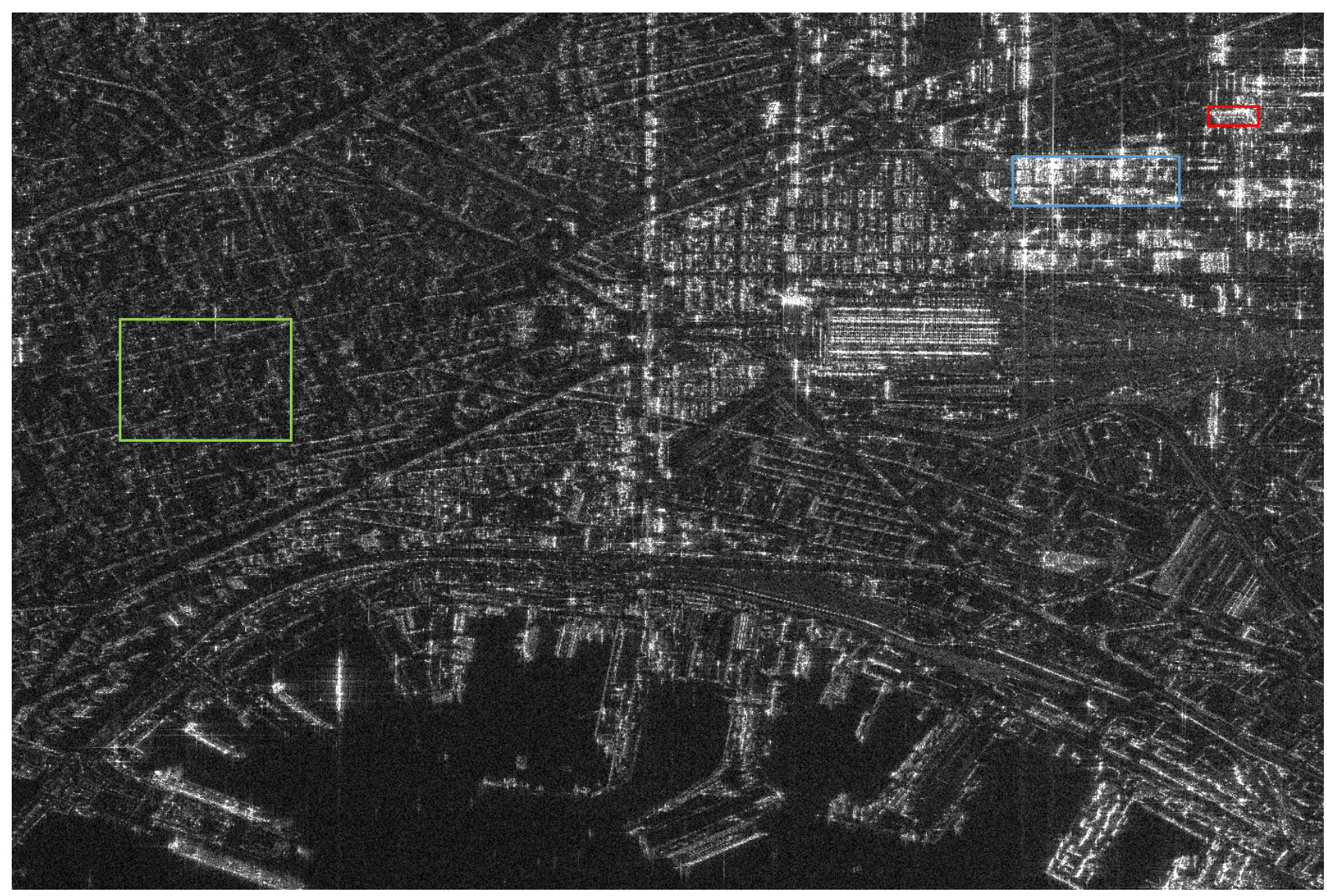

Fig. 2 Subset of the COSMO/SkyMed enhanced spotlight image containing the three considered study areas, the Forcella subset marked in green, the business district subset in blue, and the courthouse building layover in red. Near range is on the left.

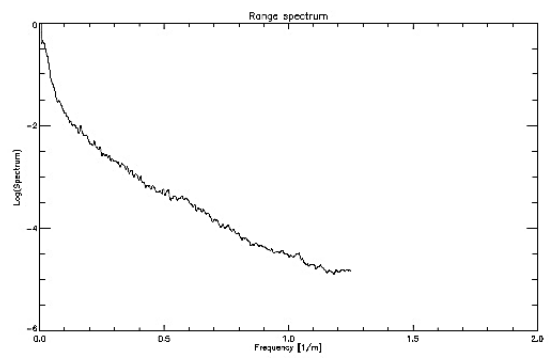

(a)

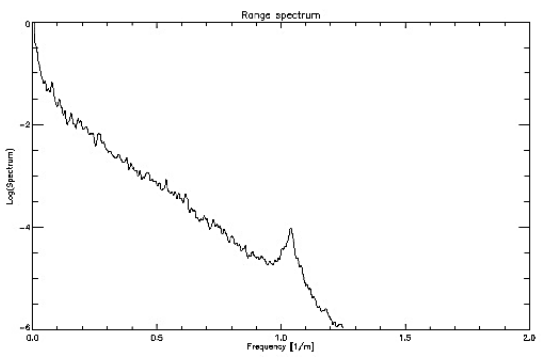

(b)

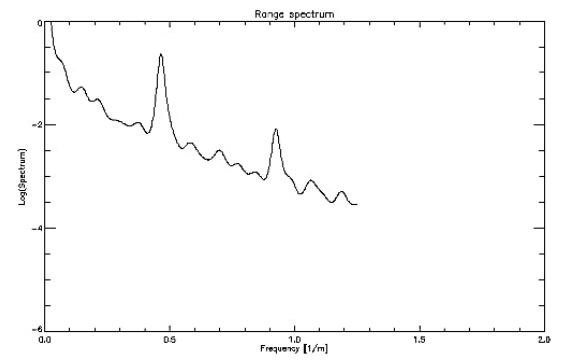

(c)

Fig. 3 Logarithmic plots of the range PSD of the three selected subsets: Forcella (a), business district (b), courthouse (c).

the presence of the periodic structure related to the building floors is evident in this case.

\section{PARAMETER RETRIEVAL}

In this section we explore the potentialities of the proposed model for the extraction of relevant physical parameters of the observed urban scene. The first proposed application regards the estimation of building floor heights. In particular, if we consider a portion of an image containing a building façade, then the positions of peaks in the range profile PSD can be used to retrieve the floor height, as suggested also in [3]. In particular, using the result in (4) to evaluate the slantrange period $d$ and converting it in a vertical period by dividing it by $\cos (\vartheta)$, where $\vartheta$ is the incidence angle, we can compute the floor height as

$$
h_{\text {floor }}=\frac{d}{\cos \vartheta}=\frac{2 \pi}{\eta_{1} \cos \vartheta} .
$$


It can be shown that the relative height precision, which for this spectral-based method depends on the resolution of the considered spectral estimator [5], is of the order of the ratio of floor height and profile length, and the absolute height precision can be smaller or even much smaller than geometric resolution. For instance, we can consider the image of the building layover of Fig. 2, whose estimated PSD is reported in Fig. 3 (c). A Google SketchUp 3D model is available for this building and is reported in Fig. 4 (a), while in Fig. 4 (b) the layover extracted from the considered image is shown. Periodic features related to the presence of the floor pattern are clearly visible on the image. From the estimated PSDs, we evaluate the wavenumber location of the peaks present in the appropriate range of wavenumbers typical of realistic floor heights. Therefore, through (5) we can get an estimate of the floor height, using the appropriate value of the incidence angle (i.e., $49.5^{\circ}$ ). The obtained result for $h_{\text {floor }}$ is $3.31 \mathrm{~m}$ and is very close to the value estimated from the available 3D model, i.e. $3.3 \mathrm{~m}$.

Another potential application is relevant to the case in which the bright spots in the image are due to distributed targets or groups of very close pointlike scatterers. In this case, as noted in Section 2, the amplitude image PSD tends to be well approximated by an exponential function, and its logarithmic plot is approximated by a straight line, where the exponent of the PSD, and hence the slope of its logarithmic plot, is related to the size of the area covered by the group of scatterers. For the considered image, we estimated the value of the exponent through linear regression on the logarithmic plot of the PSD of the subset relevant to the business district shown in Fig. 3 (b): the obtained value is -3.59 , i.e. the width covered by the group of pulses is equal to about $1.8 \mathrm{~m}$ in slant range. Subtracting the slant range resolution $a=0.679 \mathrm{~m}$, we obtain that the size of the area covered by the group of scatterers is equal to about $1.1 \mathrm{~m}$. This value is lower for the case of the Forcella subset, where the exponent of the PSD shown in Fig. 3 (a) is equal to -2.96 , implying a width occupied by the group of pulses of $1.48 \mathrm{~m}$ in slant range, and a size of the area covered by the group of scatterers of about $0.8 \mathrm{~m}$. These result, although preliminary, seem very promising and demonstrate that this parameter can be potentially used to characterize urban areas presenting different geometric (e.g. orientation with respect to the line of flight, wall depth) and electromagnetic features (e.g. complex dielectric constant).

\section{CONCLUSIONS}

In this paper, a new stochastic model for VHR SAR amplitude (or intensity) images of urban areas has been presented. The model has been obtained by schematizing a VHR SAR amplitude image as a collection of spatially randomly distributed, sparse brilliant points, or lines, over a dark background, and possibly (if building façades are present and dominant) of bright features forming periodic spatial patterns. The proposed model has been verified using one Cosmo/SkyMed enhanced spotlight image of the area of Naples, Italy. The potentiality of the model in supporting the extraction of some relevant scene parameters from measured PSDs has been also discussed. As first examples, we have considered the retrieval of building floor heights and of the average range size of small distributed targets.

\section{ACKNOWLEGEMENT}

This work was supported by ASI in the framework of the AO project "Buildings feature extraction from single SAR images: application to Cosmo/SkyMed high resolution SAR images."

\section{REFERENCES}

[1] G. Franceschetti, A. Iodice, and D.Riccio, "A Canonical Problem in Electromagnetic Backscattering from Buildings", IEEE Trans. Geosci. Remote Sens., GE-40, pp. 1787- 1801, Aug. 2002.

[2] R. Guida, A. Iodice, D. Riccio, and U. Stilla, "Model-Based Interpretation of High-Resolution SAR Images of Buildings", IEEE J. Sel. Topics Appl. Earth Observ., vol. 1, no. 2, pp. 107-119, June 2008.

[3] G. Di Martino, A. Iodice, D. Riccio, and G. Ruello, "A New Algorithm for Building Feature Extraction from Single Amplitude SAR Images", Proceedings IGARSS 2013, Melbourne (Australia), July 2013.

[4] S. Auer, C. Gisinger, and R. Bamler, "Characterization of SAR image patterns pertinent to individual façades", in Proc. IEEE Int. Geosci. Remote Sens. Symp., pp. 3611-3614, July 2012.

[5] S. M. Kay, Modern Spectral Analysis. Englewood Cliffs, NJ: Patience Hall, 1999.

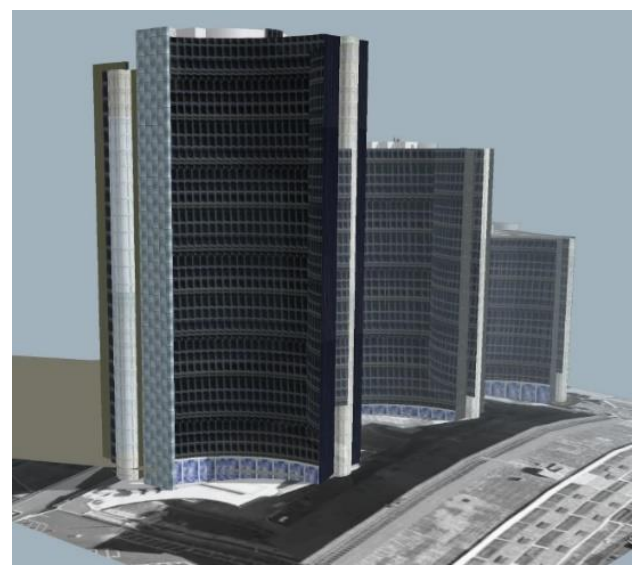

(a)

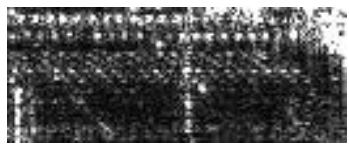

(b)

Fig. 4 Google SketchUp 3D model of the courthouse (a) and its layover extracted from the amplitude SAR image (b). 